\title{
The risk of pancreatitis with sitagliptin therapy in older adults: a population-based cohort study
}

\author{
Kristin K. Clemens MD, Eric McArthur MSc, Jamie L. Fleet BHSc, Irene Hramiak MD, \\ Amit X. Garg MD PhD
}

\section{Abstract}

Background: The risk of pancreatitis with sitagliptin use in routine care remains to be established in older patients. We aimed to determine this risk in older adults who were newly prescribed sitagliptin versus an alternative hypoglycemic agent in the outpatient setting.

\begin{abstract}
Methods: In a population-based retrospective cohort study in Ontario from 2010 until 2012 involving adults aged 66 years and older, we studied those who were newly prescribed sitagliptin or an alternative hypoglycemic agent. Our primary outcome of interest was a hospital encounter (emergency department visit or hospital admission) with acute pancreatitis within 90 days. We used inverse probability of treatment weighting to balance the 2 groups and logistic regression with a robust variance estimate to calculate odds ratios (ORs) and 95\% confidence intervals (Cls).

Results: A total of 57689 patients (mean age 74 yr) were newly prescribed sitagliptin, and 83405 patients (mean age 75 yr) were given an alternative hypoglycemic agent (metformin, glyburide, gliclazide or insulin) during the study period. After weighting, there were no significant differences in measured baseline characteristics between groups. In the weighted sample, sitagliptin was not associated with an increased risk of a hospital encounter with pancreatitis compared with alternative hypoglycemic agents (weighted total 46 of 57689 patients taking sitagliptin [0.08\%] v. 48 of 55705 patients taking alternative hypoglycemic agents [0.09\%], absolute risk difference $-0.01 \%$ [95\% $\mathrm{Cl}-0.05 \%$ to $0.02 \%$ ], OR 0.92 [95\% $\mathrm{Cl} 0.55$ to 1.55$]$ ).
\end{abstract}

Interpretation: Older adults newly prescribed sitagliptin in routine care were not at a substantially higher risk of pancreatitis than those prescribed alternative hypoglycemic agents. These findings are reassuring for those who use or prescribe sitagliptin in the management of type 2 diabetes.

itagliptin, a dipeptidyl-peptidase-4 (DPP-4) inhibitor, reduces blood glucose by decreasing glucagon secretion and blocking the breakdown of glucagon-like peptide-1, an incretin hormone that stimulates insulin secretion in a glucose-dependent fashion. ${ }^{1}$ Sitagliptin was the first DPP-4 inhibitor approved on the Ontario's general benefits formulary in June of 2010. Because of its relative potency (it decreases glycosylated hemoglobin by up to $1 \%$ as monotherapy) and low risk of hypoglycemia, ${ }^{2}$ sitagliptin use has increased substantially over recent years (more than 700000 prescriptions in Ontario from June 2010 to June 2012). ${ }^{3}$

Despite its benefits, sitagliptin has been linked with pancreatitis in case reports, animal studies and postmarketing drug surveillance studies. ${ }^{4-7}$ It has been postulated that incretin drugs (including DPP-4 inhibitors and glucagon-like peptide-1 agonists) might promote pancreatitis by increasing pancreatic mass, modifying enzyme secretion, disturbing acinar architecture, promoting inflammation, or increasing ductal turnover and metaplasia. ${ }^{6,8}$ As pancreatitis can lead to morbidity and mortality, warnings of the association have been published by regulatory agencies, pharmaceutical companies and diabetes association guidelines (Appendix 1, available at www.cmajopen.ca/content/3/2/E172/suppl/DC1).

Competing interests: Unrelated to this project, Irene Hramiak's institution received research funding from AstraZeneca/Bristol-Myers Squibb, Eli Lilly, Janssen-Ortho/Johnson \& Johnson, Merck, Novo Nordisk, Pfizer and Sanofi-Aventis. She has been a board member for AstraZeneca/Bristol-Myers Squibb, Eli Lilly, Janssen-Ortho/Johnson \& Johnson, Merck, Novo Nordisk, Pfizer, Sanofi-Aventis, Abbott, Boehringer Ingelheim, GlaxoSmithKline and Medtronic. Irene Hramiak has also received payment for lectures from AstraZeneca/Bristol-Myers Squibb, Eli Lilly, Merck and Novo Nordisk. Amit Garg received an investigator-initiated grant from Astellas Pharma and Roche to support a Canadian Institutes of Health Research study in living kidney donors, and his institution received unrestricted research funding unrelated to this project from Pfizer. No other competing interests were declared.

This article has been peer reviewed.

Correspondence to: Kristin Clemens, kclemens2008@meds.uwo.ca

CMAJ Open 2015. DOI:10.9778/cmajo.20140060 
In real-practice observational studies, however, the link between DPP-4 inhibitors and pancreatitis has been inconsistently described, and studies have been limited in their collection of baseline covariates, drug use and health care use. ${ }^{5,7,9}$ Studies have also used self-reported outcomes, ${ }^{7,9,10}$ and have been limited to younger populations, making results less generalizable to older adults. ${ }^{5,11} \mathrm{We}$ thus aimed to examine the risk of acute pancreatitis with sitagliptin therapy in routine care in a large, representative population of older adults.

\section{Methods}

\section{Study design and setting}

We conducted a population-based retrospective cohort study involving adults aged 66 years and older from June 2010 to December 2012 using linked (via unique, encoded identifiers) health care databases in Ontario, Canada. Ontario has about 1.8 million adults aged 65 years and older who have comprehensive universal health care. This includes coverage for outpatient prescription medications, physician services, hospital admissions and diagnostic tests. ${ }^{12}$

This study was conducted at the Institute for Clinical Evaluative Sciences according to a prespecified protocol that was approved by the research ethics board at Sunnybrook Health Sciences Centre (Toronto, Canada). Informed consent was not required. Our report follows guidelines for the description of observational studies. ${ }^{13}$

\section{Data sources}

We obtained patient characteristics, drug use, covariate information and outcome data using records from 6 databases. Vital statistics were obtained from the Registered Persons Database of Ontario, which holds demographic information on all residents who have been issued a health card. The Ontario Drug Benefit Program database contains accurate records of all drug formulary medications dispensed to those aged 65 years and older, with an error rate of less than $1 \% .{ }^{14}$ Diagnostic and procedural information on hospital admissions and emergency department visits was abstracted from the Canadian Institute for Health Information's Discharge Abstract Database and the National Ambulatory Care Reporting System database, respectively. Covariate information was derived from the Ontario Health Insurance Plan database, which includes health claims for inpatient and outpatient physician services. We used the Institute for Clinical Evaluative Sciences Physician Database to determine prescriber information. Previous studies have used these databases to research adverse drug events and outcomes. ${ }^{15-19}$ A subpopulation in southwestern Ontario had outpatient glycosylated hemoglobin measurements available before a new hypoglycemic agent prescription. ${ }^{20}$

With the exception of prescriber information (missing in about $9.6 \%$ ) and income quintile (missing in about $0.4 \%$ ), databases were complete for all variables used. International Statistical Classification of Diseases and Related Health Problems, 10th revision (post-2002) and Canadian Classification of Health Interventions (post-2002) codes were used to assess baseline comorbidities and investigations in the 5 years before the hypoglycemic agent prescription (Appendix 2, available at www.cmajopen.ca/content/3/2/E172/suppl/DC1). Codes used to assess the outcome of acute pancreatitis are detailed in Appendix 3 (available at www.cmajopen.ca/content/3/2/E172/ suppl/DC1).

\section{Patient selection}

To mimic routine practice, we studied older adults newly prescribed sitagliptin or an alternative hypoglycemic agent (metformin, glyburide, gliclazide or insulin) between June 2010 (when sitagliptin was first openly listed on the province's formulary) and December 2012. The date of the new hypoglycemic drug prescription served as the index date (cohort entry date or start time for follow-up).

In the sitagliptin group we excluded the following patients: those in their first year of eligibility for prescription drug coverage (aged $65 \mathrm{yr}$ ) to avoid incomplete medication records; those with a hospital discharge in the 2 days before or on the index date to ensure these were new sitagliptin prescriptions (in Ontario, patients continuing a medication initiated in hospital typically have their medication dispensed on the same day or the day after discharge); those with a code for anesthesia or an epidural in the 30 days before the index date to exclude those with a recent surgery, a risk factor for pancreatitis; those with evidence of a pancreas transplant or pancreatectomy in the 5 years before the index date, to exclude those with surgical manipulation of the pancreas; those with a prescription for 1 or more DPP-4 inhibitors in the 1 year prior (to define new use); and those prescribed saxagliptin or a sitagliptin-metformin combination pill (to restrict to sitagliptin use only).

In patients prescribed an alternate hypoglycemic agent, we applied similar exclusion criteria with differences as follows. We excluded those initiated on metformin without evidence of a code for diabetes in the Ontario Diabetes Database, ${ }^{21}$ because diabetes itself is a risk factor for pancreatitis, and metformin can be prescribed for indications other than diabetes; ;,7 those with a prescription for the same hypoglycemic agent in the 1 year prior (to define new use); those with a prescription for a DPP-4 inhibitor in the 1 year prior (to compare mutually exclusive groups); and those who were previously entered in the sitagliptin cohort. A patient could enter the cohort only once.

\section{Exposure}

For the primary analysis, we used an "intention to treat" exposure definition. Following a new hypoglycemic agent prescription, patients were followed until they experienced the primary outcome (defined below), reached 90 days of follow-up or died.

\section{Outcomes}

The primary outcome was a hospital encounter (emergency department visit or hospital admission) with acute pancreatitis within 90 days of the index date. We chose 90 days of follow-up to avoid crossover in drug therapy that could occur with longer periods of follow-up, because prescriptions covered by Ontario's drug plan are prescribed at no more than 100-day intervals, and because previous reports have suggested that patients may be at risk for pancreatitis within this time frame of study. ${ }^{6,11,22}$ 


\section{Statistical analysis}

We used standardized differences to compare baseline characteristics between groups. This metric describes differences between group means relative to the pooled standard deviation and is considered meaningful if greater than $10 \% .^{23}$

The propensity score was derived from a logistic regression model with 29 baseline covariates incorporated into the score based on prior recommended methods (Appendix 4, available at www.cmajopen.ca/content/3/2/E172/suppl/DC1). ${ }^{24}$ Inverse probability of treatment weights were calculated using the propensity model to create a sample in which the distribution of measured baseline covariates was independent of treatment assignment. $^{24}$

For the reference group, we considered those prescribed an alternative hypoglycemic agent. We expressed the risk of acute pancreatitis in relative and absolute terms. To calculate odds ratios (ORs) and 95\% confidence intervals (CIs), we fit a logistic regression model using a robust variance estimate, accounting for inverse probability of treatment weights.

We conducted all analyses with SAS version 9.3 including analyses we undertook after knowledge of the primary results. We interpreted 2 -tailed $p$ values less than 0.05 as significant.

\section{Sensitivity analyses}

In time-to-event analysis we extended follow-up beyond 90 days, terminating the observation period for reasons of death, discontinuation of the study hypoglycemic agent, receipt of a nonstudy hypoglycemic agent or the last date of available

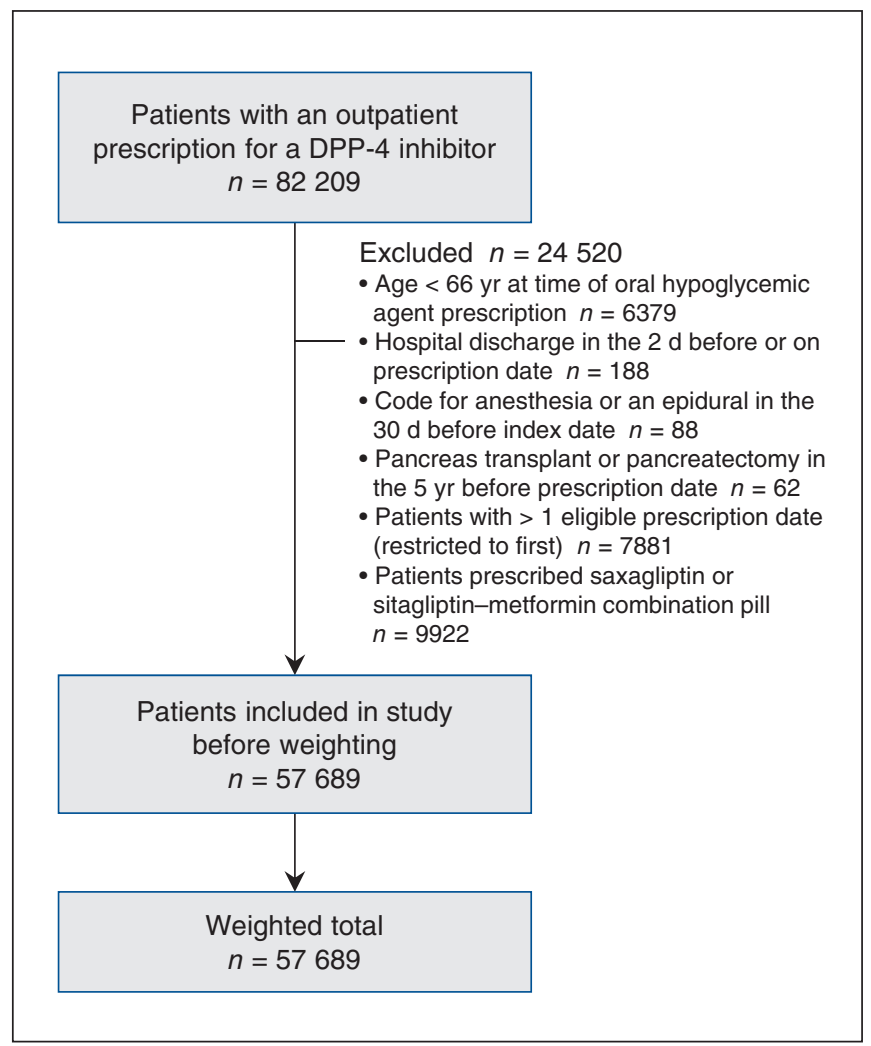

Figure 1: Flow diagram representing patient selection in the sitagliptin cohort. Note: DPP-4 = dipeptidyl-peptidase-4. records (Mar. 31, 2013). We also restricted our cohort to patients accrued after June 2011 to address the possibility of incomplete DPP-4 inhibitor records before June 2010, because sitagliptin became available on the general access formulary in June 2010. Differences in diagnostic testing between groups were evaluated over the course of follow-up by investigating the proportion of patients with amylase and lipase tests. Finally, we examined separately the risk of pancreatitis in patients taking metformin, sulfonylurea and insulin, and in those where the relevant hypoglycemic agent was prescribed as monotherapy or in the setting of another hypoglycemic agent or agents.

\section{Results}

\section{Baseline characteristics}

Patient selection is presented in Figures 1 and 2. There were 57689 patients newly prescribed sitagliptin and 83405 patients newly prescribed an alternative hypoglycemic agent over the course of study. Before weighting, patients given sitagliptin were younger and taking more medications, and in the years prior, had fewer hospital visits and more diagnostic testing than patients given alternative hypoglycemic agents. After weighting, there were 57689 in the sitagliptin group and 55

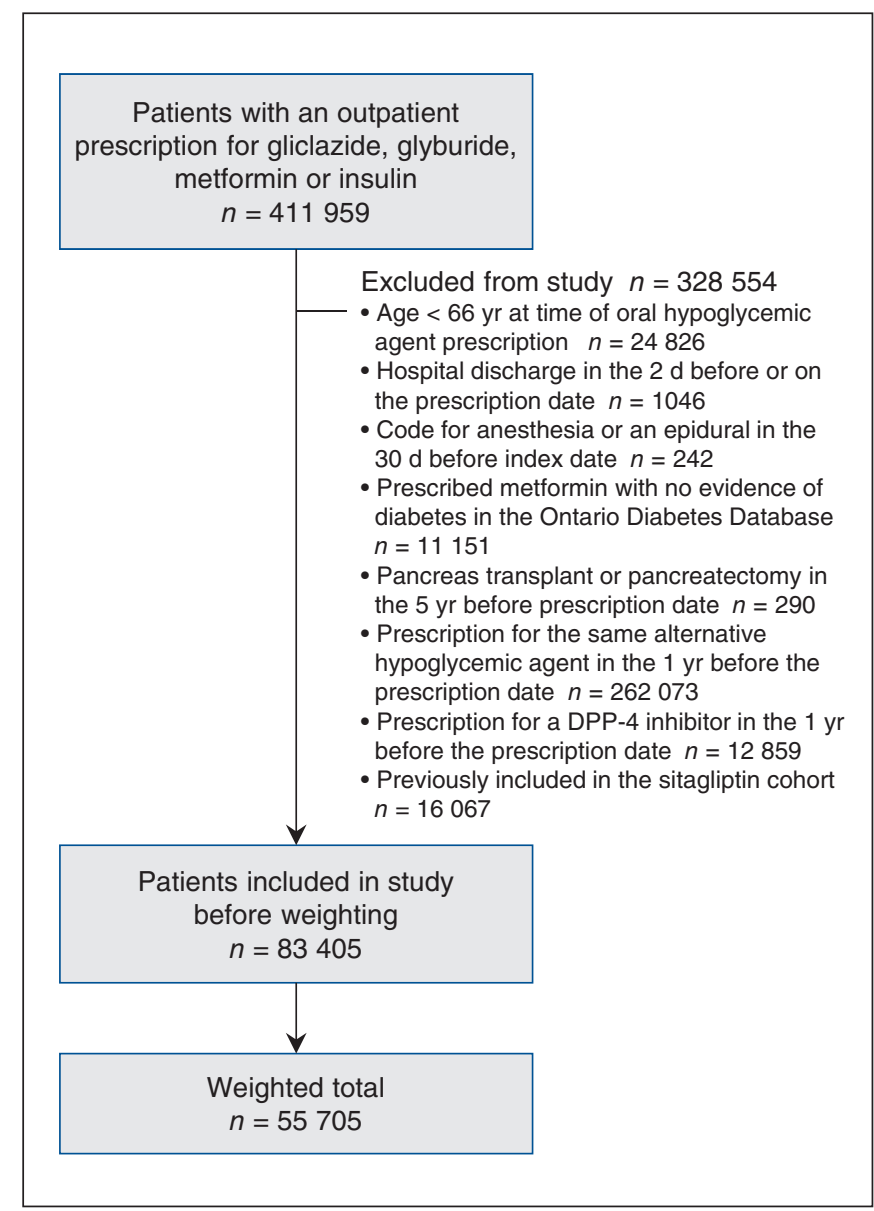

Figure 2: Flow diagram representing patient selection in the alternative hypoglycemic agent cohort. Note: DPP-4 = dipeptidyl-peptidase-4. 
705 in the alternative hypoglycemic agent group, and characteristics were similar between the groups (standardized difference less than 10\%). Baseline characteristics are outlined in Tables 1-3 and Appendix 5 (available at www.cmajopen.ca/ content/3/2/E172/suppl/DC1).

Beyond the index prescription, $84.7 \%$ and $76.1 \%$ of patients given sitagliptin and alternative hypoglycemic agents, respectively, filled at least 1 additional prescription for the relevant hypoglycemic agent. The duration of continuous study drug use (defined by the duration of 1 prescription overlap- ping with a subsequent prescription, allowing for a 10-day grace period between prescriptions), was about $81,64,91,82$ and 108 days in the sitagliptin, insulin, glyburide, gliclazide and metformin groups, respectively.

\section{Outcomes}

Results for the primary outcome of pancreatitis are presented in Table 4. Sitagliptin use was not associated with a higher 90-day risk of a hospital encounter with pancreatitis than alternative hypoglycemic agent use (weighted total 46 of 57

\section{Table 1: Characteristics in patients given sitagliptin or an alternative hypoglycemic agent, before and after propensity weighting}

\begin{tabular}{|c|c|c|c|c|c|c|}
\hline \multirow[b]{3}{*}{ Characteristic } & \multicolumn{6}{|c|}{ No. $(\%)$ of patients* } \\
\hline & \multicolumn{3}{|c|}{ Before weighting } & \multicolumn{3}{|c|}{ After weighting $†$} \\
\hline & $\begin{array}{c}\text { Sitagliptin } \\
n=57689\end{array}$ & $\begin{array}{c}\text { Alternative } \\
\text { hypoglycemic agent } \\
n=83405\end{array}$ & $\begin{array}{l}\text { Standardized } \\
\text { difference, \%‡ }\end{array}$ & $\begin{array}{c}\text { Sitagliptin } \\
n=57689\end{array}$ & $\begin{array}{c}\text { Alternative } \\
\text { hypoglycemic } \\
\text { agent } \\
n=55705\end{array}$ & $\begin{array}{r}\text { Standardized } \\
\text { difference, \%‡ }\end{array}$ \\
\hline \multicolumn{7}{|l|}{ Age at index date, yr } \\
\hline Mean & 73.98 & 75.07 & 17 & 73.98 & 74.07 & 2 \\
\hline Median & 73 & 74 & & 73 & 73 & \\
\hline Standard deviation & 6.25 & 6.93 & & 6.25 & 5.20 & \\
\hline $66-70$ & $21105(36.58)$ & 26880 (32.23) & 9 & 21105 (36.58) & $20171(36.21)$ & 1 \\
\hline $71-75$ & $15829(27.44)$ & $21282(25.52)$ & 4 & $15829(27.44)$ & $15199(27.28)$ & 0 \\
\hline $76-80$ & $11228(19.46)$ & 16397 (19.66) & 0 & $11228(19.46)$ & $10661(19.14)$ & 1 \\
\hline $81-85$ & $6431(11.15)$ & $11112(13.32)$ & 7 & $6431(11.15)$ & $6350(11.40)$ & 1 \\
\hline $86-90$ & 2461 (4.27) & 5828 (6.99) & 12 & 2461 (4.27) & $2678 \quad(4.81)$ & 3 \\
\hline$>90$ & $635(1.10)$ & 1906 (2.29) & 9 & $635(1.10)$ & $646 \quad(1.16)$ & 1 \\
\hline Female sex & $27584(47.82)$ & 40312 (48.33) & 1 & $27584(47.82)$ & 26279 (47.18) & 1 \\
\hline Rural location & $5997(10.40)$ & $12396(14.86)$ & 13 & $5997(10.40)$ & 6275 (11.26) & 3 \\
\hline Long-term care facility & $1446(2.51)$ & $5581 \quad(6.69)$ & 20 & $1446(2.51)$ & 1594 & 2 \\
\hline \multicolumn{7}{|l|}{ Income quintile§ } \\
\hline 1 & $12582(21.81)$ & $18233(21.86)$ & 0 & $12582(21.81)$ & 12447 (22.34) & 1 \\
\hline 2 & $13048(22.62)$ & $18029(21.62)$ & 2 & $13048(22.62)$ & $12134(21.78)$ & 2 \\
\hline 3 & 11601 (20.11) & $16572(19.87)$ & 1 & $11601(20.11)$ & $11150(20.02)$ & 0 \\
\hline 4 & $10860(18.83)$ & $16320(19.57)$ & 2 & $10860(18.83)$ & $10845(19.47)$ & 2 \\
\hline 5 & 9419 (16.33) & $13878(16.64)$ & 1 & 9419 (16.33) & 8894 (15.97) & 1 \\
\hline Missing & $179(0.31)$ & $373(0.45)$ & 2 & $179(0.31)$ & $234 \quad(0.42)$ & 2 \\
\hline \multicolumn{7}{|l|}{ Prescribing physician } \\
\hline Endocrinology & 4813 (8.34) & 3042 (3.65) & 20 & 4813 (8.34) & $5047 \quad(9.06)$ & 3 \\
\hline General practice & 42925 (74.41) & $66305(79.50)$ & 12 & $42925(74.41)$ & 40948 (73.51) & 2 \\
\hline Internal medicine & $2454(4.25)$ & $2281 \quad(2.73)$ & 8 & $2454(4.25)$ & $2407 \quad(4.32)$ & 0 \\
\hline Nephrology & $342(0.59)$ & $778 \quad(0.93)$ & 4 & $342(0.59)$ & $360 \quad(0.65)$ & 1 \\
\hline Other & $1830(3.17)$ & 2252 (2.70) & 3 & 1830 & $1727 \quad(3.10)$ & 0 \\
\hline Missing & 5325 (9.23) & $8169 \quad(9.79)$ & 2 & 5325 (9.23) & $5216 \quad(9.36)$ & 0 \\
\hline $\begin{array}{l}\text { *Unless stated otherwise. } \\
\text { †All patients identified before } \\
\text { †Standardized differences ar } \\
\text { pooled standard deviation; a } \\
\text { §Income was categorized int }\end{array}$ & $\begin{array}{l}\text { e weighting were inc } \\
\text { re less sensitive to } s \\
\text { value greater than } \\
\text { to fifths of average } n\end{array}$ & the analyses. The numb & f patients indicat & $\begin{array}{l}\text { epresents a weigh } \\
\text { a measure of the } \\
\text { e groups. }\end{array}$ & $\begin{array}{l}\text { tal. } \\
\text { ence between groups }\end{array}$ & ided by the \\
\hline
\end{tabular}


689 patients taking sitagliptin [0.08\%] v. 48 of 55705 patients taking alternative hypoglycemic agents [0.09\%], absolute risk difference $-0.01 \%$ [95\% CI $-0.05 \%$ to $0.02 \%$ ], OR 0.92 [95\% CI 0.55 to 1.55$])$.

\section{Sensitivity analyses}

Our findings remained robust in sensitivity analyses. Results of the time to event analysis are shown in Table 5. Sitagliptin use was not associated with a higher risk of a hospital encounter

Table 2: Comorbidities in patients given sitagliptin or an alternative hypoglycemic agent, before and after propensity weighting

\begin{tabular}{|c|c|c|c|c|c|c|}
\hline \multirow[b]{3}{*}{ Comorbidities* } & \multicolumn{6}{|c|}{ No. (\%) of patients† } \\
\hline & \multicolumn{3}{|c|}{ Before weighting } & \multicolumn{3}{|c|}{ After weighting $\ddagger$} \\
\hline & $\begin{array}{c}\text { Sitagliptin } \\
n=57689\end{array}$ & $\begin{array}{c}\text { Alternative } \\
\text { hypoglycemic } \\
\text { agent } \\
n=83405\end{array}$ & $\begin{array}{l}\text { Standardized } \\
\text { difference, \%§ }\end{array}$ & \multicolumn{2}{|r|}{$\begin{array}{c}\text { Alternative } \\
\text { hypoglycemic } \\
\text { agent } \\
n=55705\end{array}$} & \multirow{2}{*}{$\begin{array}{c}\text { Standardized } \\
\text { difference, \%§} \\
0\end{array}$} \\
\hline Gallstones/biliary stones & 2163 (3.75) & 3152 (3.78) & 0 & 2163 (3.75) & $2059 \quad(3.70)$ & \\
\hline Calcium disorder & $123(0.21)$ & $273(0.33)$ & 2 & $123(0.21)$ & $154 \quad(0.28)$ & 1 \\
\hline Alcoholism & $240(0.42)$ & $659(0.79)$ & 5 & $240 \quad(0.42)$ & $338 \quad(0.61)$ & 3 \\
\hline Tobacco use & $3128(5.42)$ & $4662(5.59)$ & 1 & $3128 \quad(5.42)$ & $3222 \quad(5.78)$ & 2 \\
\hline Pancreatic neoplasm & $101 \quad(0.18)$ & $250 \quad(0.30)$ & 3 & $101 \quad(0.18)$ & $178 \quad(0.32)$ & 3 \\
\hline $\begin{array}{l}\text { Endoscopic retrograde } \\
\text { cholangiopancreatography }\end{array}$ & $281(0.49)$ & $561 \quad(0.67)$ & 2 & $281 \quad(0.49)$ & $334 \quad(0.60)$ & 2 \\
\hline Chronic kidney diseaseף & $6069(10.52)$ & $10321(12.37)$ & 6 & $6069(10.52)$ & $6714(12.05)$ & 5 \\
\hline Bile duct neoplasm & $118(0.20)$ & $272(0.33)$ & 2 & $118 \quad(0.20)$ & $158 \quad(0.28)$ & 2 \\
\hline HIV & $50(0.09)$ & $67(0.08)$ & 0 & $50 \quad(0.09)$ & $33 \quad(0.06)$ & 1 \\
\hline $\begin{array}{l}\text { Systemic lupus } \\
\text { erythematosus }\end{array}$ & 739 (1.28) & 1107 & 0 & $739 \quad(1.28)$ & $659 \quad(1.18)$ & 1 \\
\hline Polyarteritis nodosa & $216 \quad(0.37)$ & $429(0.51)$ & 2 & $216 \quad(0.37)$ & $248 \quad(0.45)$ & 1 \\
\hline Celiac disease & $78 \quad(0.14)$ & $158(0.19)$ & 1 & $78 \quad(0.14)$ & $103(0.18)$ & 1 \\
\hline Obesity & $4219 \quad(7.31)$ & 5468 (6.56) & 3 & $4219 \quad(7.31)$ & $3696 \quad(6.63)$ & 3 \\
\hline \multicolumn{7}{|l|}{ Charlson comorbidity index ${ }^{* *}$} \\
\hline Mean & 1.13 & 1.22 & 5 & 1.13 & 1.23 & 7 \\
\hline $0-1$ & $40624(70.42)$ & $57156(68.53)$ & 4 & $40624(70.42)$ & $37816(67.89)$ & 5 \\
\hline 2 & $7861(13.63)$ & $10430(12.51)$ & 3 & 7861 (13.63) & $8053(14.46)$ & 2 \\
\hline$>3$ & 9204 (15.95) & $15819(18.97)$ & 8 & $9204(15.95)$ & $9836(17.66)$ & 5 \\
\hline Diabetic retinopathy & $636 \quad(1.10)$ & $842(1.01)$ & 1 & $636 \quad(1.10)$ & $783 \quad(1.41)$ & 3 \\
\hline Diabetic neuropathy & $576 \quad(1.00)$ & $843 \quad(1.01)$ & 0 & $576 \quad(1.00)$ & $597 \quad(1.07)$ & 1 \\
\hline Peripheral vascular disease & $679 \quad(1.18)$ & 1259 & 3 & $679 \quad(1.18)$ & $740 \quad(1.33)$ & 1 \\
\hline Heart failure & $6606(11.45)$ & $11932(14.31)$ & 9 & $6606(11.45)$ & 7068 (12.69) & 4 \\
\hline Coronary artery bypass graft & 1766 (3.06) & 2553 (3.06) & 0 & $1766 \quad(3.06)$ & $1781 \quad(3.20)$ & 1 \\
\hline Hypertension & $49934(86.56)$ & $64828(77.73)$ & 23 & $49934(86.56)$ & $48277(86.67)$ & 0 \\
\hline Coronary artery disease & $16299(28.25)$ & $23740(28.46)$ & 0 & $16299(28.25)$ & $16144(28.98)$ & 2 \\
\hline Myocardial infarction & $1243(2.15)$ & 2479 (2.97) & 5 & $1243 \quad(2.15)$ & $1381 \quad(2.48)$ & 2 \\
\hline $\begin{array}{l}\text { Stroke/transient ischemic } \\
\text { attack }\end{array}$ & 1377 (2.39) & 3011 & 7 & 1377 (2.39) & 1535 (2.76) & 2 \\
\hline Dialysis & 1251 & 2992 (3.59) & 8 & $1251 \quad(2.17)$ & $1545 \quad(2.77)$ & 4 \\
\hline Renal transplant & $30(0.05)$ & $118 \quad(0.14)$ & 3 & $30 \quad(0.05)$ & $62 \quad(0.11)$ & 2 \\
\hline Hypoglycemia & $770 \quad(1.33)$ & $1354 \quad(1.62)$ & 2 & $770 \quad(1.33)$ & $818 \quad(1.47)$ & 1 \\
\hline Acute or chronic pancreatitis & $216 \quad(0.37)$ & $467(0.56)$ & 3 & $216 \quad(0.37)$ & $267 \quad(0.48)$ & 2 \\
\hline Hyperglycemic emergency & $117(0.20)$ & $245 \quad(0.29)$ & 2 & $117(0.20)$ & $162(0.29)$ & 2 \\
\hline $\begin{array}{l}{ }^{*} \text { Comorbidities were assessed by ad } \\
\text { †Unless stated otherwise. } \\
\text { †All patients identified before weighti } \\
\text { §Standardized differences are less } \mathrm{s} \\
\text { pooled standard deviation; a value gr } \\
\text { ๆWe identified individuals with chron } \\
\text { a median estimated glomerular filtrat } \\
\text { min per } 1.73 \mathrm{~m}^{2} \text { (interquartile range } 5 \\
{ }^{*} \text { Charlson comorbidity index was ca }\end{array}$ & $\begin{array}{l}\text { ministrative database } \\
\text { ng were included in t } \\
\text { ensitive to sample siz } \\
\text { eater than } 10 \% \text { is int } \\
\text { c kidney disease usir } \\
\text { on rate (eGFR) of } 38 \\
6 \text { to } 82 \text { ). See Fleet e }\end{array}$ & $\begin{array}{l}\text { in the previous } 5 \text { ye } \\
\text { halyses. The number of } \\
\text { in traditional hypothesi } \\
\text { ted as a meaningful di } \\
\text { validated algorithm of } \\
\text { min per } 1.73 \mathrm{~m}^{2} \text { (interq }\end{array}$ & $\begin{array}{l}\text { atients indicated rep } \\
\text { tests. They provide a } \\
\text { arence between the } \\
\text { annosis and physicia } \\
\text { artile range } 27 \text { to } 52 \text { ) }\end{array}$ & $\begin{array}{l}\text { sents a weighted tota } \\
\text { easure of the differen } \\
\text { ups. } \\
\text { laim codes. In Ontar } \\
\text { s absence identifies }\end{array}$ & $\begin{array}{l}\text { eetween groups divic } \\
\text { is algorithm identifie } \\
\text { ents with a median e }\end{array}$ & $\begin{array}{l}\text { ided by the } \\
\text { es patients with } \\
\text { GFR of } 69 \mathrm{~mL} /\end{array}$ \\
\hline
\end{tabular}


with pancreatitis than alternative hypoglycemic agent use (Cox proportional hazards model with inverse probability of treatment weights; hazard ratio [HR] 1.18 [95\% CI 0.94 to 1.49]. The analysis of patients newly prescribed sitagliptin or an alternative hypoglycemic agent after June 2011 produced results similar to our primary analysis (Appendix 6, available at www.
cmajopen.ca/content/3/2/E172/suppl/DC1). Similar proportions of amylase and lipase tests were noted in the 2 groups (Appendix 7, available at www.cmajopen.ca/content/3/2/E172/ suppl/DC1). Appendices 7-9 (available at www.cmajopen.ca/ content/3/2/E172/suppl/DC1) illustrate similar low event rates across groups of study.

Table 3 (part 1 of 2): Medication and laboratory data in patients given sitagliptin or an alternative hypoglycemic agent, before and after propensity weighting

No. $(\%)$ of patients*

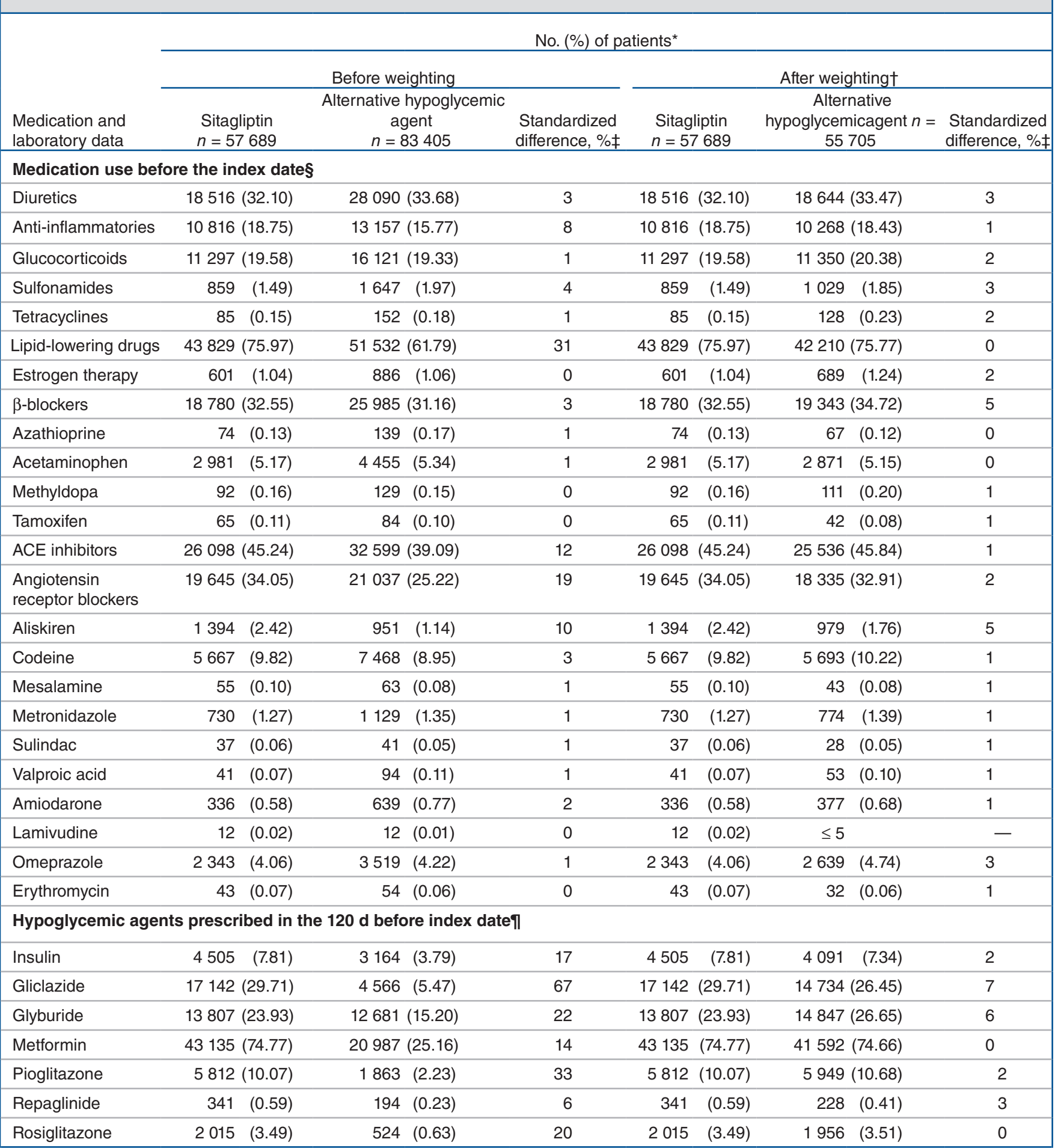




\section{OPEN}

Research

\section{Interpretation}

In our cohort, the initiation of sitagliptin was not associated with a higher 90-day risk of a hospital encounter with acute pancreatitis than the initiation of metformin, glyburide, gliclazide or insulin in routine care.

The findings of our study are consistent with the results of randomized controlled trials. A recent systematic review and meta-analysis of 55 randomized trials (33 350 patients) found no difference in the risk of pancreatitis between patients prescribed incretin drugs versus patients prescribed a placebo, lifestyle or other oral hypoglycemic agents (OR 1.11, 95\% CI 0.57 to 2.17). ${ }^{27} \mathrm{~A}$ cardiovascular study of patients randomly assigned to saxagliptin or placebo found that rates of acute pancreatitis were similar in both groups (22 of 8280 patients given saxagliptin $[0.3 \%]$ v. 16 of 8212 patients given placebo [0.2\%], $p=0.42) .{ }^{28}$

Table 3 (part 2 of 2): Medication and laboratory data in patients given sitagliptin or an alternative hypoglycemic agent, before and after propensity weighting

No. $(\%)$ of patients ${ }^{*}$

\begin{tabular}{|c|c|c|c|c|c|c|}
\hline \multirow[b]{3}{*}{$\begin{array}{l}\text { Medication and } \\
\text { laboratory data }\end{array}$} & \multicolumn{6}{|c|}{ No. $(\%)$ of patients* } \\
\hline & \multicolumn{3}{|c|}{ Before weighting } & \multicolumn{3}{|c|}{ After weightingt } \\
\hline & $\begin{array}{l}\text { Sitagliptin } \\
n=57689\end{array}$ & $\begin{array}{c}\text { Alternative hypoglycemic } \\
\text { agent } n=83405\end{array}$ & $\begin{array}{c}\text { Standardized } \\
\text { difference, \% }\end{array}$ & $\begin{array}{c}\text { Sitagliptin } \\
n=57689\end{array}$ & $\begin{array}{c}\text { Alternative hypoglycemic } \\
\text { agent }=55705\end{array}$ & $\begin{array}{l}\text { Standardized } \\
\text { difference, \%‡ }\end{array}$ \\
\hline \multicolumn{7}{|c|}{ Hypoglycemic agents prescribed on the index date ${ }^{\star *}$} \\
\hline Insulin & $1010 \quad(1.75)$ & $\leq 5 \ddagger \ddagger$ & - & $(1.75)$ & 0 & 19 \\
\hline Gliclazide & $6232(10.80)$ & 5578 (6.69) & 15 & $6232(10.80)$ & 0 & 49 \\
\hline Glyburide & $2982(5.17)$ & 6198 (7.43) & 9 & $2982 \quad(5.17)$ & 0 & 33 \\
\hline Metformin & $14174(24.57)$ & 1324 (1.59) & 73 & 14174 (24.57) & 0 & 81 \\
\hline Pioglitazone & $652 \quad(1.13)$ & 409 (0.49) & 7 & $652 \quad(1.13)$ & $771 \quad(1.38)$ & 2 \\
\hline Repaglinide & $61 \quad(0.11)$ & $27 \quad(0.03)$ & 3 & $61 \quad(0.11)$ & $13 \quad(0.02)$ & 3 \\
\hline Rosiglitazone & $89 \quad(0.15)$ & $51 \quad(0.06)$ & 3 & $89 \quad(0.15)$ & $105 \quad(0.19)$ & 1 \\
\hline \multicolumn{7}{|c|}{ Hypoglycemic agents prescribed in the $1 \mathrm{yr}$ to $120 \mathrm{~d}$ before the index date $t$} \\
\hline Insulin & $4671 \quad(8.10)$ & $5272(6.32)$ & 37 & $4671 \quad(8.10)$ & $4213(7.56)$ & 2 \\
\hline Gliclazide & $17175(29.77)$ & $5886 \quad(7.06)$ & 61 & $17175(29.77)$ & $14249(25.58)$ & 9 \\
\hline Glyburide & $17038(29.53)$ & $15101(18.11)$ & 27 & $17038(29.53)$ & $17053(30.61)$ & 2 \\
\hline Metformin & $45376(78.66)$ & $27777(33.30)$ & 103 & 45376 (78.66) & $41580(74.64)$ & 9 \\
\hline Pioglitazone & $7023(12.17)$ & 2918 (3.50) & 33 & $7023(12.17)$ & $6493(11.66)$ & 2 \\
\hline Repaglinide & $450 \quad(0.78)$ & $372(0.45)$ & 4 & $450 \quad(0.78)$ & $353(0.63)$ & 2 \\
\hline Rosiglitazone & $2981 \quad(5.17)$ & $1123 \quad(1.35)$ & 22 & $2981 \quad(5.17)$ & 2447 (4.39) & 4 \\
\hline Recent test for $\mathrm{g}$ & lated hemoglobir & levels & & $16413(28.45)$ & $14837(26.63)$ & 4 \\
\hline \multicolumn{7}{|c|}{ Glycosylated hemoglobin level, \% } \\
\hline Mean & & & & 7.7 & 7.8 & 8 \\
\hline Median & & & & 7.4 & 7.5 & \\
\hline 25th percentile & & & & 6.9 & 6.9 & \\
\hline 75th percentile & & & & 8.2 & 8.4 & \\
\hline Standard devia & & & & 1.3 & 1.2 & \\
\hline \multicolumn{7}{|c|}{ 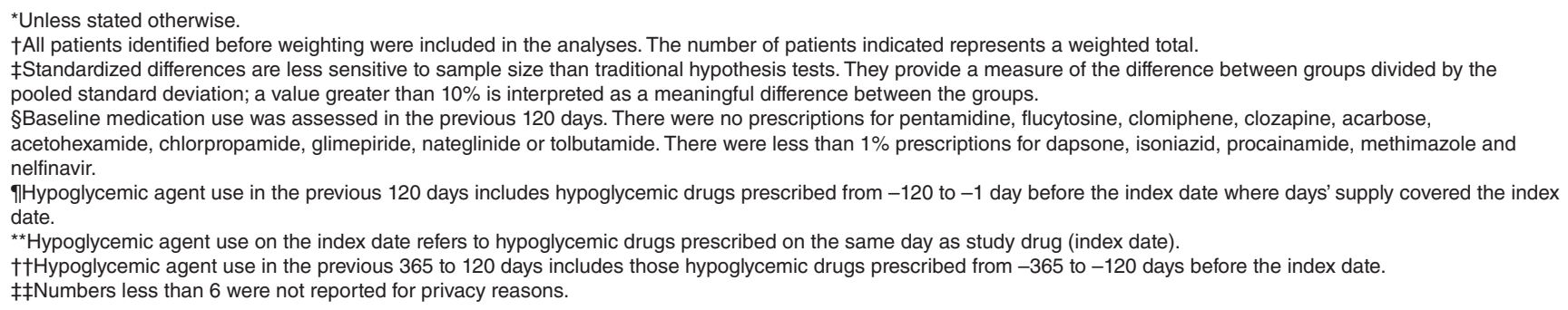 } \\
\hline
\end{tabular}


Our results are also consistent with previous observational studies. In a cohort study of 16276 patients given sitagliptin and 16281 matched patients given metformin or glyburide, the risk of acute pancreatitis was similar (relative risk 1.0, 95\% CI 0.5 to 2.0). ${ }^{29}$ An additional cohort study noted comparable rates of pancreatitis in patients taking sitagliptin and alternative hypoglycemic agents (adjusted HR 1.0, 95\% CI 0.7 to 1.3), ${ }^{5}$ as did a recent report of 20748 patients taking incretin drugs and 51712 patients taking sulfonylureas (adjusted HR $1.00,95 \%$ CI 0.59 to 1.70 ). ${ }^{30}$ Further, a study of 1003 cases and 4012 matched controls found that use of incretin drugs in the 6 months prior was not associated with pancreatitis (OR $0.98,95 \%$ CI 0.69 to 1.38$).{ }^{31}$ Similar results were noted in a recent Chinese case-control study. ${ }^{32}$

Our results, however, differ from those of published studies that report a higher risk of pancreatitis with use of DPP-4 inhibitors. Studies using the US Food and Drug Administration database have found that patients taking incretin drugs had a higher odds of pancreatitis (OR 6.74, 95\% CI 4.61 to 10.00) than patients taking other oral agents, ${ }^{7}$ and that the odds of pancreatitis in patients taking DPP-4 inhibitors was 20.8 times (95\% CI 12.6 to 34.5$)$ the odds of pancreatitis in those taking other hypoglycemic agents. ${ }^{10}$ A French surveillance study also noted that the rate of exposure to DPP-4 inhibitors was higher in cases of pancreatitis versus non-cases of pancreatitis (67 patients taking DPP-4 inhibitors in 147 cases of pancreatitis v. 421 patients taking DPP-4 inhibitors in 2962 non-cases of pancreatitis, adjusted reporting OR 12.1, $95 \%$ CI 7.3 to 20.0). ${ }^{9}$ These studies, however, may have been subject to reporting bias as events were self-reported and may have been inflated by external factors. ${ }^{7,9}$

An additional case-control study found that the adjusted odds of acute pancreatitis in those who currently (within $30 \mathrm{~d}$ ) (OR 2.24, 95\% CI 1.36 to 3.68) and who had recently used incretin drugs (past $30 \mathrm{~d}$ and less than $2 \mathrm{yr}$ ) was higher (OR $2.01,95 \%$ CI 1.37 to 3.18 ) than in those who had not used incretin drugs. ${ }^{11}$ This study performed a more limited assessment of baseline covariates and indices of health care use, and, because it was completed in a younger population (mean age $52 \mathrm{yr}$ ), may not be fully generalizable to older adults.

\section{Strengths and limitations}

Our study has several strengths. Using a large sample of older patients who used sitagliptin in a routine setting (with multiple comorbidities and taking multiple medications), our study complements information from randomized clinical trials by studying an uncommon but important adverse drug reaction. Our study has adequate statistical power, and the results are generalizable to the larger population. Our newuser design allowed us to observe outcomes after the initiation of treatment. Whereas previous studies included self-reported pancreatitis, in our study pancreatitis was documented in hospital records by the treating health care team. Additionally, to echo routine care and make our findings interpretable in clinical practice, we studied patients who were newly prescribed hypoglycemic alternatives to sitagliptin (metformin, a sulfonylurea or insulin) as a comparison group. We also carried out numerous sensitivity analyses and our primary results remained robust.

Our study has some limitations. Prospective data collection with independent outcome adjudication is a preferred methodology to a retrospective database study. We were unable to detect asymptomatic pancreatitis or pancreatitis that did not result in a hospital presentation. We accurately ascertained medications dispensed but had no information on medication use (although we note that most patients filled at least 1 additional prescription for the relevant study drug).

Given the low event rate of pancreatitis in both groups, we were able to rule out a greater than 1.6-fold increase in the risk of pancreatitis in patients newly prescribed sitagliptin compared with patients newly prescribed alternative hypoglycemic agents with adequate statistical power (upper bound of the CI), but could not rule out a smaller increase in risk. The limited number of events also precluded meaningful subgroup analysis.

The duration of our study did not allow us to measure the long-term effects of sitagliptin. However, we feel that our 90 days follow-up period was reasonable given that the mean duration of continuous drug use ranged from 64 to 108 days, previous reports have suggested that pancreatitis can manifest early in the course of drug exposure, ${ }^{6,11,22}$ and studies to date have not noted that the risk of pancreatitis differs by exposure duration. ${ }^{27,30}$

Table 4: Ninety-day risk of a hospital encounter with acute pancreatitis in patients given sitagliptin or an alternative hypoglycemic agent, ${ }^{*}$ before and after propensity weighting

\begin{tabular}{|c|c|c|c|c|c|c|}
\hline \multirow[b]{3}{*}{ Variable } & \multicolumn{4}{|c|}{ No. (\%) of events } & \multirow[b]{3}{*}{$\begin{array}{l}\text { Absolute risk } \\
\text { difference, } \\
\%(95 \% \mathrm{Cl})\end{array}$} & \multirow[b]{3}{*}{$\begin{array}{l}\text { Odds ratio } \\
(95 \% \mathrm{Cl})\end{array}$} \\
\hline & \multicolumn{2}{|c|}{ Before weighting } & \multicolumn{2}{|c|}{ After weighting $†$} & & \\
\hline & $\begin{array}{l}\text { Sitagliptin } \\
n=57689\end{array}$ & $\begin{array}{c}\text { Alternative } \\
\text { hypoglycemic } \\
\text { agent } \\
n=83405\end{array}$ & $\begin{array}{c}\text { Sitagliptin } \\
n=57689\end{array}$ & $\begin{array}{c}\text { Alternative } \\
\text { hypoglycemic } \\
\text { agent } \\
n=55705\end{array}$ & & \\
\hline Hospital encounter with acute pancreatitis & $46(0.08)$ & $83.40(0.10)$ & $46(0.08)$ & $48 \quad(0.09)$ & $\begin{array}{c}-0.01 \\
(-0.05 \text { to } 0.02)\end{array}$ & $\begin{array}{c}0.92 \\
(0.55 \text { to } 1.55)\end{array}$ \\
\hline $\begin{array}{l}\text { Note: } \mathrm{Cl}=\text { confidence interval. } \\
\text { *Patients prescribed glyburide, gliclazide, metformi }\end{array}$ & ulin served & reference grou & 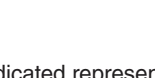 & 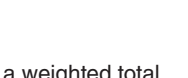 & & \\
\hline
\end{tabular}




\begin{tabular}{|c|c|c|}
\hline \multicolumn{3}{|c|}{ Table 5: Time-to-event analysis } \\
\hline \multirow[b]{2}{*}{ Variable } & \multicolumn{2}{|c|}{ No. $(\%)$ of events, ${ }^{*}$ after weighting } \\
\hline & $\begin{array}{l}\text { Sitagliptin } \\
n=57689\end{array}$ & $\begin{array}{c}\text { Alternative } \\
\text { hypoglycemic agent } \\
n=55705\end{array}$ \\
\hline $\begin{array}{l}\text { Person-years of } \\
\text { follow-up }\end{array}$ & 14988 & 16972 \\
\hline $\begin{array}{l}\text { Days of follow-up, } \\
\text { median (IQR) }\end{array}$ & $65(30-125)$ & $75(30-129)$ \\
\hline \multicolumn{3}{|l|}{ Censoring events } \\
\hline $\begin{array}{l}\text { Hospital encounters with } \\
\text { pancreatitis }\end{array}$ & $260(0.45)$ & $224(0.40)$ \\
\hline Hazard ratio $(95 \% \mathrm{Cl})$ & $1.18(0.94$ to 1.49$)$ & 1.00 (reference) \\
\hline $\begin{array}{l}\text { Event rate per } 1000 \\
\text { person-years }\end{array}$ & 17.35 & 13.18 \\
\hline \multicolumn{3}{|l|}{ Censoring events } \\
\hline Death & $9(0.02)$ & $20.95(0.04)$ \\
\hline $\begin{array}{l}\text { Study hypoglycemic } \\
\text { agent discontinued }\end{array}$ & 8579 (14.87) & 17485 (31.39) \\
\hline $\begin{array}{l}\text { Prescription for a } \\
\text { nonstudy hypoglycemic } \\
\text { agent }\end{array}$ & 48841 (84.66) & 37976 (68.17) \\
\hline
\end{tabular}

The potential for confounding and bias warrants attention. In the current study, we had limited information on factors such as obesity, body mass index and smoking status, which are known to influence the risk of pancreatitis. It is also possible that physicians may have selectively prescribed sitagliptin therapy to healthier people and may have differentially monitored patients for outcomes. However, using propensity score weighting, we obtained good balance on a large number of measured baseline characteristics between the 2 groups. Further, we note that diagnostic testing for pancreatitis was similar among the 2 groups during follow-up.

\section{Conclusion}

In older adults, the initiation of sitagliptin did not result in a higher risk of a hospital encounter with pancreatitis than initiation of an alternative diabetic medication (any of metformin, glyburide, gliclazide or insulin). These findings are reassuring for those who use or prescribe sitagliptin in the management of type 2 diabetes.

\section{References}

1. Engel SS, Williams-Herman DE, Golm GT, et al. Sitagliptin: review of preclinical and clinical data regarding incidence of pancreatitis. Int 7 Clin Pract 2010;64:984-90.

2. Fanuvia: stitagliptin tablets [product monograph]. Kirkland (QC): Merck Canada Inc.; 2013. Available: www.merck.ca/assets/en/pdf/products/JANUVIA-PM_E.pdf (accessed 2014 Mar. 13).

3. IMS/brogan [homepage on the Internet].2014. Available: www.imshealth.com/ portal/site/imshealth? CURRENT_LOCALE=en_ca (accessed 2014 Mar. 13).

4. Sue M, Yoshihara A, Kuboki K, et al. A case of severe acute necrotizing pancreatitis after administration of sitagliptin. Clin Med Insights Case Rep. 2013;6:23-7.
5. Garg R, Chen W, Pendergrass $M$. Acute pancreatitis in type 2 diabetes treated with exenatide or sitagliptin: a retrospective observational pharmacy claims analysis. Diabetes Care 2010;33:2349-54.

6. Matveyenko AV, Dry S, Cox HI, et al. Beneficial endocrine but adverse exocrine effects of sitagliptin in the human islet amyloid polypeptide transgenic rat model of type 2 diabetes: interactions with metformin. Diabetes 2009; 58:1604-15.

7. Elashoff M, Matveyenko AV, Gier B, et al. Pancreatitis, pancreatic, and thyroid cancer with glucagon-like peptide-1-based therapies. Gastroenterology 2011;141:150-6.

8. Koehler JA, Baggio LL, Lamont BJ, et al. Glucagon-like peptide-1 receptor activation modulates pancreatitis-associated gene expression but does not modify the susceptibility to experimental pancreatitis in mice. Diabetes 2009; 58:2148-61.

9. Faillie JL, Babai S, Crepin S, et al. Pancreatitis associated with the use of GLP-1 analogs and DPP-4 inhibitors: a case/non-case study from the French Pharmacovigilance Database. Acta Diabetol 2014;51:491-7.

10. Moore T, Cohen M, Furberg C. ISMP QuarterWatch: perspectives on GLP-1 agents for diabetes. 2013. Available: www.ismp.org/quarterwatch/pdfs/2012Q3. pdf. (accessed 2014 Apr. 11).

11. Singh S, Chang HY, Richards TM, et al. Glucagonlike peptide 1-based therapies and risk of hospitalization for acute pancreatitis in type 2 diabetes mellitus: a population-based matched case-control study. 7AMA Intern Med 2013;173:534-9.

12. Bronskill S, Carter M, Costa A, et al. Aging in Ontario: an ICES chartbook of bealth service use by older adults. Toronto: Institute for Clinical Evaluative Sciences; 2010. Available: www.ices.on.ca/Publications/Atlases-and-Reports/2010 /Aging-in-Ontario.aspx. (accessed 2014 Mar. 13).

13. von Elm E, Altman DG, Egger M, et al. The strengthening the reporting of observational studies in epidemiology (STROBE) statement: guidelines for reporting observational studies. Prev Med 2007;45:247-51.

14. Levy AR, O'Brien BJ, Sellors C, et al. Coding accuracy of administrative drug claims in the Ontario drug benefit database. Can 7 Clin Pharmacol 2003; 10:67-71.

15. Patel AM, Shariff S, Bailey DG, et al. Statin toxicity from macrolide antibiotic coprescription: a population-based cohort study. Ann Intern Med 2013; 158:869-76.

16. Siddiqui NF, Coca SG, Devereaux PJ, et al. Secular trends in acute dialysis after elective major surgery - 1995 to 2009. CMA7 2012;184:1237-45.

17. Shih AW, Weir MA, Clemens KK, et al. Oral bisphosphonate use in the elderly is not associated with acute kidney injury. Kidney Int 2012;82:903-8.

18. Zhao YY, Weir MA, Manno M, et al. New fibrate use and acute renal outcomes in elderly adults: a population-based study. Ann Intern Med 2012; 156:560-9.

19. Gandhi S, Fleet JL, Bailey DG, et al. Calcium-channel blocker-clarithromycin drug interactions and acute kidney injury. FAMA 2013;310:2544-53.

20. Gandhi S, Shariff SZ, Beyea MM, et al. Identifying geographical regions serviced by hospitals to assess laboratory-based outcomes. BMF Open 2013;3: pii:e001921.

21. Hux JE, Ivis F, Flintoft V, et al. Diabetes in Ontario: determination of prevalence and incidence using a validated administrative data algorithm. Diabetes Care 2002;25:512-6.

22. Information for Healthcare Professionals - acute pancreatitis and sitagliptin (marketed as Fanuvia and Fanumet). Silver Spring (MD): U.S. Food and Drug Administration; 2009. Available: www.fda.gov/Drugs/DrugSafety/PostmarketDrug SafetyInformationforPatientsandProviders/DrugSafetyInformationforHeathcare Professionals/ucm183764.htm. (accessed 2014 Mar. 13).

23. Austin PC. Using the standardized difference to compare the prevalence of a binary variable between two groups in observational studies. Commun Stat Simulat 2009;38:1228-34.

24. Austin PC. An introduction to propensity score methods for reducing the effects of confounding in observational studies. Multivariate Bebav Res 2011;46:399-424.

25. Fleet JL, Dixon SN, Shariff SZ, et al. Detecting chronic kidney disease in population-based administrative databases using an algorithm of hospital encounter and physician claim codes. BMC Nephrol 2013;14:81.

26. Charlson ME, Pompei P, Ales KL, et al. A new method for classifying prognostic comorbidity in longitudinal studies: development and validation. $\mathcal{F}$ Chronic Dis 1987;40:373-83.

27. Li L, Shen J, Bala MM, et al. Incretin treatment and risk of pancreatitis in patients with type 2 diabetes mellitus: systematic review and meta-analysis of randomized and non-randomised studies. BM7 2014;348:g2366.

28. Scirica BM, Bhatt DL, Braunwald E, et al. Saxagliptin and cardiovascular outcomes in patients with type 2 diabetes mellitus. NEngl f Med 2013;369:1317-26.

29. Dore DD, Seeger JD, Arnold Chan K. Use of a claims-based active drug safety surveillance system to assess the risk of acute pancreatitis with exenatide or sitagliptin compared to metformin or glyburide. Curr Med Res Opin 2009;25: 1019-27.

30. Faillie JL, Azoulay L, Patenaude V, et al. Incretin based drugs and risk of acute pancreatitis in patients with type 2 diabetes: cohort study. BMF 2014; 348:g2780.

31. Giorda CB, Picariello R, Nada E, et al. Incretin therapies and risk of hospital 
admission for acute pancreatitis in an unselected population of European patients with type 2 diabetes: a case-control study. Lancet Diabetes Endocrinol 2014;2:111-5.

32. Chou HC, Chen WW, Hsiao FY. Acute pancreatitis in patients with type 2 diabetes mellitus treated with dipeptidyl peptidase-4 inhibitors: a populationbased nested case-control study. Drug Saf 2014;37:521-8.

Affiliations: Department of Medicine (Clemens, Fleet, Garg) Western University, London, Ont.; Institute for Clinical Evaluative Sciences (McArthur, Fleet, Garg), Toronto, Ont.; Division of Endocrinology and Metabolism (Hramiak), Department of Medicine, Western University, London, Ont.; Department of Epidemiology and Biostatistics (Garg), Western University, London, Ont.

Contributors: Kristin Clemens contributed to the study design and interpretation of the results. Eric McArthur contributed to the study design, acquired the data and carried out the statistical analysis. Jamie Fleet contributed to the study design. Irene Hramiak contributed to the study concept and interpretation of the results. Amit Garg contributed to the study concept, the study design and interpretation of the results. Kristin Clemens and Amit Garg contributed to drafting the manuscript, which Eric McArthur, Jamie Fleet and Irene Hramiak revised. All of the authors approved the final version of the manuscript to be submitted, and agreed to act as guarantors of the work.
Funding: This project was conducted at the Institute for Clinical Evaluative Sciences (ICES) Western. The Institute for Clinical Evaluative Sciences is funded by an annual grant from the Ontario Ministry of Health and Long-term Care. Core funding for ICES Western is provided by the Academic Medical Organization of Southwestern Ontario, the Schulich School of Medicine and Dentistry, Western University and the Lawson Health Research Institute. The sponsors had no role in the design and conduct of the study; collection, management, analysis, and interpretation of the data; preparation, review, or approval of the manuscript; or decision to submit the manuscript for publication. The opinions, results, and conclusions are those of the authors, and no endorsement by the sponsors is intended or should be inferred.

Acknowledgements: The authors thank Brogan Inc., Ottawa, for use of its drug product and therapeutic class database; Gamma-Dynacare for use of the outpatient laboratory database; the team at London Health Sciences Centre, St Joseph's Health Care; the Thames Valley Hospitals for providing access to the Cerner laboratory database; and Salimah Shariff, $\mathrm{PhD}$ (from the Institute for Clinical Evaluative Sciences Western), for administrative support.

Supplemental information: For reviewer comments and the original submission of this manuscript, see www.cmajopen.ca/content/3/2/E172/ suppl/DC1 